\title{
Hormonal and Genetic Role Affecting the Bone Resorption in Patients with Osteoporosis
}

\author{
Susi R. Puspitadewi \\ Postgraduate Program \\ Faculty of Dentistry, Universitas Indonesia, \\ Jakarta, Indonesia \\ susi.puspita@yahoo.com
}

\author{
Elza I. Auerkari \\ Department of Oral Biology \\ Faculty of Dentistry, Universitas Indonesia, \\ Jakarta, Indonesia \\ eauerkari@yahoo.com
}

\begin{abstract}
Increasing age, especially in postmenopausal women, shows a decline in anabolic hormones activity and an increase in anti-anabolic hormones activity. This causes bone resorption and may lead to osteoporosis. Osteoporosis is a degenerative disorder marked by decrease in bone mass because of imbalance between bone resorption and formation. Bone strength is determined by morphology, bone structure \& geometry, bone remodelling and quality of extracellular matrix. The balance in bone resorption and formation determines bone density and affects one's susceptibility to bone fracture. Bone resorption is affected by bone metabolism process on cellular level, and on edentulous areas is more affected by hormonal, nutritional and metabolism factors. Morrison and Coll have found that there are many polymorphisms in osteoporosis etiopathogenesis, some among them showing a large influence on BMD (Bone mineral density) compared to others, including Estrogen, VDR, COLIA1, PTH, PTHR1, and encoding genes for some cytokines and growth factors.
\end{abstract}

Keywords-osteoporosis, bone, resorption, hormone

\section{INTRODUCTION}

Increasing age, especially in postmenopausal women, shows a decline in anabolic hormones activity and an increase in anti-anabolic hormones activity. This causes bone resorption and may lead to osteoporosis. Osteoporosis is one of the degenerative disorders characterized by a decrease in bone mass due to an imbalance between resorption and bone formation [1,2]. Currently osteoporosis is seen as a heterogeneous state that can occur at any age due to endocrine, metabolic, genetic and various mechanical stress factors[3]. Osteoporosis results in reduced bone mass due to imbalances of resorption and bone formation that may be physiologic or pathologic [4]. Changes bone density of the jaw is more common in people with osteoporosis compared with normal people. The upper jawbone is more susceptible to osteoporosis than the lower jawbone $[5,6]$. The deficiency of the estrogen hormone affects the loss of bone mass of the jaw. This condition has received attention in the field of dentistry because of the increasing number of osteoporosis patients from year to year. Symptoms of osteoporosis usually begin to arise in women aged 51-75 years [7]. Loss of bone mineral content in the oral cavity is closely related to age factors that appear significantly in middle age or the elderly and are associated with physiological and sex conditions. Women at high risk of osteoporosis after menopause due to cessation of estrogen production.

The loss of bone mineral is two times greater in women than in men. Women lose $1-5 \%$ bone mass during the first year at the beginning of menopause, then bone mass disappears slowly. The condition of osteoporosis and bone loss in the oral cavity is an asymptomatic symptom, usually only known after the onset of bone fractures and tooth mobillity [8,9]. Physiologically, the risk of missing teeth becomes higher with advancing age, increasing the need for dental prostheses. The percentage of the female population using dental prosthesis is higher than that of the male population [10]. This indicates that a relatively high number of women in elderly populations will need dental replacement. Missing teeth will affect the stomatognatic system, including the chewing or masticating function, and it may also be related to systemic disruption due to decreasing intake of nutrition [11]. For older people this is an important issue. Furthermore, the maintenance of dentures or dental prosthesis requires support of the jawbone. This support is not available in persons with osteoporosis [12].

Genetic factor plays an important role in osteoporosis, in which fenotypes also play a role on bone mineral density (BMD) [3]. From the study on twin families, about $50-85 \%$ variation on bone mass is determined genetically. Unfortunately, there is little data available that can explain osteoporotic fracture caused by genetic factor because of the high cost needed and difficulties in obtaining adequate samples. Some studies showed that familial history of fracture is a high risk factor of bone fracture, independent of bone mineral density. Two other importante factors that contribute to bone fragility because of osteoporosis are aging factor and decrease in hormonal functions [6]. Strong evidences show that a decrease in hormonal functions, especially in estrogen secretion on postmenopausal women, lead to an increase in bone resorption rate [13].

The balance in bone resorption and formation determines bone density and affects one's susceptibility to bone fracture. Strong evidences show that a decline in endocrine functions, especially estrogen secretion in menopausal women, results in increase of bone 
resorption rate. One of the factors affecting bone resorption is interaction between anabolic hormones (estrogen \& testosterone) and anti-anabolic hormones (adrenal, glocorticoid and hidrocortison) [14]. Postmenopausal women will have an increase in follicle stimulating hormone (FSH) levels up to 10-20 times and increase in luteinizing hormone (LH) levels up to 3 times normal values and then reaches the highest level after 1-3 years post menoapuse, which shows ovarium failure because of the change of ovarium stromal cells into mesenchymal tissues, therefore reducing the ability of ovarium to produce steroid hormone [15]. Decline of ovarium functions physiologically causes changes on some body organs like bones and cardiovascular system. These changes are associated with hypoestrogenemia. One of the changes from hypoestrogenemia on postmenopausal women is an increase in bone resorption but no increase in bone formation [16].

\section{A. Bone cells}

\section{LITERATURE REVIEW}

Bone is a dynamic tissue which has complex cellular regenerative system [3]. Bone metabolism is regulated by bone cells (Osteoblasts, Osteoclasts and Osteocytes), which respond to various stimuli including chemical, mechanical, electrical and magnetic stimuli. Specific stimuli are regulated by cell receptors that are found on the cell membrane or inside the cell [7]. The receptors in the cell membrane bind to the stimuli from the outside and transmit the information to the cell nucleus through the transduction mechanism. Meanwhile, cell receptors (in the cytoplasm or nucleus) bind to hormone stimuli (usually steroid hormones) that pass through the cell membrane and into the cell to transfer the effector to the nucleus in which there is a steroid receptor complex bound to a specific deoxyribonucleic acid (DNA) of the gene sequence [12]. One of the factors that affect bone metabolism is the interaction of hormones anabolism (estrogen and testosterone) and hormones antianabolism (adrenal, glocorticoid and hydrocortisone). The process of bone metabolism is governed by the relationship that occurs between hormones and other factors. Among them are Fibroblast Growth Factors (FGF) which can improve the proliferation of osteoblasts and the synthesis of collagen tissue in bone. FGF is a powerful activator [14]. Insulin-like growth factors (IGF, type 1 and 2) or somatomedin are other factors that can increase amount of protein from osteoid by promoting preosteoblast proliferation by reducing collagen degradation followed by increased protein synthesis [18].

\section{B. Bone romedelling process}

The process of bone remodeling is a process of bone resorption and new bone formation that results in growth and replacement of bone. Remodeling is a replacement of old / damaged bone that begins with bone resorption by osteoclasts followed by bone formation by osteoblasts [13]. Both bone formation and bone resorption are balanced, meaning the bone resorbed will be followed by the formation of bones in exactly the same amount, this is called coupling. Remodeling usually takes place after the age of 30 years. This process lasts until the age of about 40 years. The end result of bone remodeling is the preservation of mineralized bone matrix and collagen. This process lasts until the age of about 40 years. The end result of bone remodeling is the preservation of mineralized bone matrix and collagen [19]. The process of bone remodeling is regulated by a number of hormones and other local factors. Hormones that promote bone remodeling are parathyroid hormone (PTH), thyroxine, Growth Hormone, vitamin D, and inhibited by calcitonin, estrogen, glucocorticoids, sex hormones and thyroid hormones [20]. Glucocorticoids have the effect of stimulating bone resorption, through decreased calcium absorption followed by increased PTH [11].
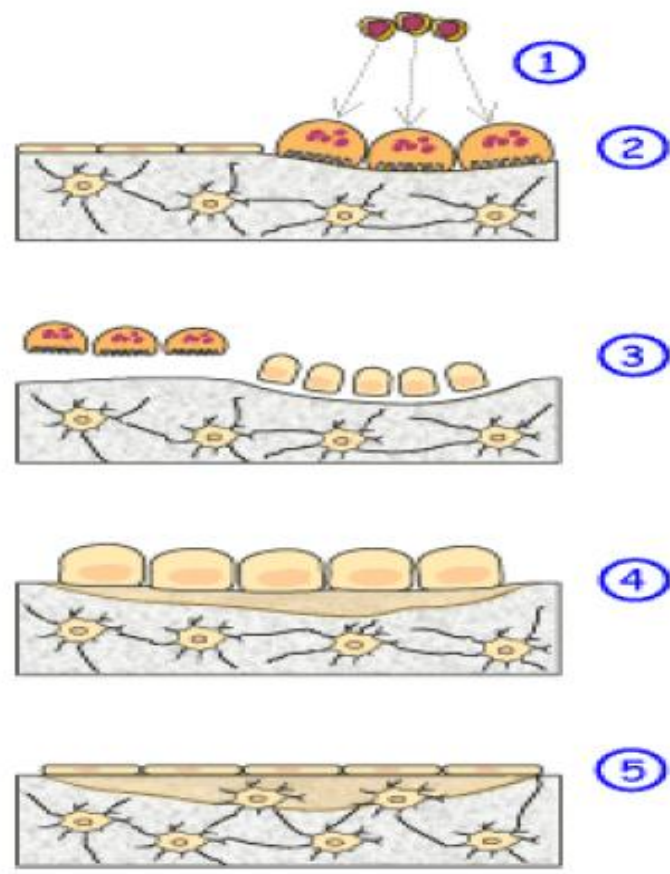

Figure 1. Bone remodeling process [13].

Bone remodeling begins with the activation of osteoblasts in the bone surface and bone marrow stromal cells [14]. This event is followed by a signal directed to recruit osteoclasts from the hemopoiesis system to ensure the activity of bone resorption. After the bone is resorbed by the osteoclast, the matrix component consisting of TGF $\beta$, IGF I (insulin like growth factor), collagen, osteoclasine, and other protein and mineral components will be released into the microenvironment. The free GF at resorption also plays a role in recruiting new osteoblasts, which begin with the process of collagen synthesis and biomineralization [12]. Bone remodelling process follows some phases 1). Activation pre-osteoclasts are stimulated into mature, active osteoclasts. 2). Resorption osteoclasts digest older bone matrix. 3). Reversal end of resorption, osteoclasts are replaced by osteoblasts. 4). Formation 
osteoblasts produce new bone matrix. 5). Passive phase osteoblasts finish matrix production and be embedded in it (Figure 1). Some osteoblasts form a line of cells on the surface of newly formed bone.

\section{Factors which affect bone remodelling}

The process of bone metabolism is regulated by the relationship that occurs between hormones and other sistemyc factors. Among them are Fibroblast Growth Factors (FGF) which can improve the proliferation of osteoblasts and the synthesis of collagen tissue in bone. FGF is a powerful activator. Insuline-like growth factors (IGF, type 1 and 2) or somatomedin are other factors that can increase the amount of protein from osteoid by promoting preosteoblast proliferation by reducing collagen degradation followed by increased protein synthesis [16].

Changes in the form of growth factors (TGF, $\beta 1$ and $\beta 2)$ are thought to play an important role in cell maturation by stimulating precursor cells into osteoblasts and alkaline phosphatase synthesis, TGF- $\beta$ expression also associated with collagen type 1 tissue synthesis. Platelet-derived Growth Factor is also found in bone matrices that stimulate osteoprogenitor cell activity and protein formation. Colony stimulating factors (CSF) play a role in the proliferation of osteoclasts and the delivery of information between osteoclasts and osteoblasts [18].

The balance between osteoclast and osteoblast activity is affected by constant steroid hormone levels in bone cells. Disturbances in the regulation are evident in aging and estrogen 16 hormone deficiency states. In addition to age and menopause factors, risk factors that also affect bone mass and density include early bone density (which is brought at birth) and the availability of calcium. Another factor that plays a role in bone remodeling regulation is vitamin $\mathrm{D}$, where vitamin $\mathrm{D}$ supplementation is proven to increase bone density, and often occurs in menopausal women [9]. Other regulators are parathyroid hormones and various cytokines and enzymes that act as both regulators and koreseptor in differentiation as well Activity of bone cells. Parathyroid hormone can increase bone resorption by releasing calcium from the bone matrix into the blood circulation to keep blood calcium levels normalized [15].

\section{Estrogen}

The estrogen hormone plays an important role in the basic regulation of bone remodeling and therapeutic in women. Decreased estrogen may decrease osteoid matrix production, increase trabecular bone formation, and spur bone resorption processes and increase bone turnover [20]. Manolagas reported that the hormone estrogen can decrease apoptosis of osteoblast cells, prolonging the life of osteoblast cells, this is the mechanism of estrogen to control bone formation process. Chen, et al. reported that estrogen hormone affects apoptosis of osteoblasts and osteoclasts through the mechanism of phosphorylation [21]. Increased thyroid hormone concentration, prolactin hormone, growth hormone will increase IGF-1 production by osteoblasts, resulting in increased preosteoblast proliferation, protein synthesis and decreased protein degradation. Cortisol and steroids can increase bone turnover and directly stimulate the process of resorption and bone formation [22]. Estrogen can be categorized into three groups: estron (E1), estradiol (E2) and Estriol (E3) [18]. Currently there is another structure known as anti estrogen, but in the non-reproductive organs are estrogenic, these structures are called selective estrogen receptor modulators (SERMs). The main estrogen produced by the ovary is estradiol. Presently, two kinds of estrogen receptor (ER) are known, namely estrogen alpha receptor $(\mathrm{ER} \alpha)$ and estrogen beta receptor $(\mathrm{ER} \beta)$ [20]. In bone, these estrogen receptors are distributed in various cells, including osteoblasts. Estrogen is a regulator of bone growth and bone homeostasis that plays a very important role in bone metabolism, affecting the activity of osteoblasts and osteoclasts, including maintaining the balance of both cells. The estrogen indirect effects on bone are associated with calcium homeostasis which includes calcium absorption in the intestine, modulating $1,25(\mathrm{OH}) 2$ vitamin $\mathrm{D}$, calcium excretion in the kidney and parathyroid hormone secretion [21,23]. Estrogen plays a role in bone remodeling through the antiresorptive effect of bone by inhibition of cytokines. Antiresorptive effect of estrogen is mediated by estrogen receptors, one of which is estrogen receptor alpha (ESR1). This ESR1 plays a dominant role in mediating effects of estrogen on bone. ESR1 is encoded by a gene located on a $6 \mathrm{q} 25$ chromosome with 8 exons along $140 \mathrm{~kb} .5$ [22].

Shevde et al. [18] found that estrogen works by suppressing osteoclast differentiation. It has previously been explained that osteoclast formation requires an interaction between RANK (receptor activator nuclear factor kappa B, NF-кB) and its ligand, RANKL. The interaction between RANK and RANKL is regulated by osteoprotegerin (OPG) production. Estrogen controls osteoclast differentiation by inhibiting the interaction between RANK and RANKL. Estrogen may inhibit the production of IL-6, IL-1 and / or TNF- $\alpha$, IL-11, IL-7 and TGF- $\beta$ which are also important in osteoclast differentiation. Length of osteoclast life also determines the number of osteoclast cells on the surface of bone resorption.Hughes et al. ${ }^{18}$ found that estrogen can induce apoptosis and osteoclast death, therefore it can directly decrease bone resorption activity $[21,23]$.

\section{E. Cytokine}

One of the factors that influence the development and differentiation of osteoblasts and osteoclasts is cytokine and colony stimulating factor (CSF). Cytokines that can stimulate osteoclast development are IL-1, IL-3, IL-6, IL-11 and TNF- $\alpha$ acts as sensitive markers of systemic inflammation as well as releasing C-reactive protein (CRP) production in the liver [20].

Cytokines that can stimulate osteoclast development are IL-1, IL-3, IL-6, IL-11 and TNF- $\alpha$ acts as sensitive 
markers of systemic inflammation as well as releasing C-reactive protein (CRP) production in the liver ${ }^{20}$. In the case of rheumatoid arthritis, bone loss occurring in the joints and skeletal bones is caused by the release of proteinases (metalloproteinases) and proinflammatory cytokines such as IL-1, and TNF- $\alpha$ which causes destruction of cartilage and bone [24]. The activity of this disease is a risk factor for osteoporosis. Proinflammatory cytokines have the potential for bone remodeling and pathogenesis of osteoporosis. Interleukin (IL)-6 assists in osteoclast differentiation and activation. IL-1, a bone-resorbing stimulator correlates with bone loss acceleration seen in idiopathic and postmenopausal osteoporosis. Cytokines that inhibit osteoclasts are interleukins 4, 10, 18 (IL4, IL10, IL18) and interferonY [25]. IL6 is important because it has a pathogenesis role in some diseases characterized by increased bone remodeling and excessive bone resorption. IL6 is produced by stromal cells or osteoblast layers in response to stimuli by cytokines and other GFs, such as IL 1, TNF, TGF $\beta$, PDGF and IGF. By itself or in conjunction with other agents, IL6 may stimulate osteoclastogenesis and trigger bone resorption [24-27].

\section{F. Parathyroid hormone}

Parathyroid hormone is an amino acid polypeptide, which is produced by the parathyroid glands. Parathyroid gland consists of four small structures located behind the thyroid gland. Parathyroid hormone stimulates bone resorption resulting in elevated blood calcium levels [11]. Parathyroid hormone can not bind closely to receptors in osteoclasts, so it can not directly affect the behavior of osteoclasts. But this hormone can bind to receptors in the osteoblasts, which can stimulate bone formation. It has been believed that the bond between parathyroid hormone and osteoblasts results in an increase in RANKL expression, which indirectly increases osteoclast activity [17].

There are at least three kinds of PTH receptors. One of them also becomes a receptor of PTHrP (parathyroid hormone related protein) called PTH / PTHrP receptor. The second receptor PTH2 (hPTH2-R) does not bind to PTHrP, present in the brain, placenta, and pancreas. PTH works directly on the bone, improves bone resorption and mobilizes calcium [9,11]. With the exception of increasing plasma calcium, PTH also increases the excretion of phosphate in the urinary tract so as to decrease plasma phosphate levels. PTH also increases the reabsorption of calcium in the distal tubules and increases the formation of 1,25 dihydroxycolecalciferol so that it can increase the absorption of calcium from the intestine. In the long term, PTH also stimulates osteoblast and osteoclast function. The mechanism of action of PTH (Parathyroid Hormone) in its activity will use cAMP-adenylyl cyclase second messenger system [16].

In the structure of DNA, to separate individually some sequence characteristics that quantitatively and qualitatively influence gene expression. Polymorphism may involve a single or multiple nucleotide sequence (SNP), known as tandem (microsatellite), located at a particular locus [20]. In the last ten years, since studies conducted by Morrison and Coll, individually there are many polymorphisms involved in the ethiopathogenesis of osteoporosis and among them have a great influence on BMD, some of them in VDR, COLIA1, ER, CYP19, PTHR1, LRP5 and encoding genes for several cytokines and growth factors (TGF $\beta$, Activina A, IL-6 and IGF1) [23].

\section{G. 1,25 (oh) vitamin D (calcitriol)}

Vitamin D plays an important role in calcium homeostasis, in regulation of growth and differentiation of bone cells, in the intestines for calcium and phosphorus absorption, as well as PTH secretion (parathyroid hormone) [16]. Vitamin D consists of 2 substances, calcifediol (1,25-dihydroxyvitamin D) and calcitriol (1,25-trihydroxivitamin D) [2,12]. Calcitriol is synthesized in the liver and kidneys with vitamin D ingredients, from food and vitamin D3 from skin synthesis due to sun exposure. It has a VDR (Vitamin D Receptor) gene receptor which is a pathogenesis of osteoporosis, osteoarthritis, diabetes mellitus, breast and prostate cancer, and physiology in weight reduction [9]. Parathyroid hormone is needed to convert calsifediol to calsitriol because this hormone is the main stimulator of the activity of 1- $\alpha$ hydroxylase in the kidneys. Parathyroid hormone is needed to convert calsifediol to calsitriol because this hormone is the main stimulator of the activity of 1- $\alpha$ hydroxylase in the kidneys [17]. Changes of calsifediol to calsitriol play a role in the process of osteoblast maturation. Decreased concentrations of calsifediol and calsitriol are associated with increased activation of metabolic units in bone [18].

\section{H. Collagen type I alpha 1 (colla 1) gene polymorphism}

Bone structure is a mineral component $(70 \%$ of the bone dry weight), a bone organic substance consisting of bone and cell matrices. The osteoporotic bone matrix, consisting of collagen type 1 protein and noncollagen protein, constitutes $95 \%$ of the bone organic substance. Genes that encode collagen type I (COL1A 1 and 2) are very important and have been widely studied as factors that affect the pathogenesis of osteoporosis. Polymorphisms in this gene have been shown to increase their prevalence in osteoporosis patients [13].

A positive association between COL1 A1 Sp1 polymorphism and bone mass or osteoporotic fractures has been reported in some populations. Ethnic differences have also been reported, where the prevalence of COLIA1 Sp1 alleles with polymorphism is found in Caucasian but rarely in ethnic African and Chinese. The overall data suggest that COL1A1 Sp1 polymorphism causes a malfunction, some of which are in vitamin $\mathrm{D}$ receptors (VDR), type 1 collagen (COL1A1 \& 2), adverse effects on bone composition (matrix) and bone mechanical strength [23]. COL1A1 
polymorphism may not be valuable as a treatment target but as a risk marker for osteoporotic fractures [16].

\section{CYP19}

In pre-menopausal women, this gene is involved in estrogen production, in addition to estradiol, while postmenopause this hormone is produced through androgen aromatization of the extragonadal especially in adipose tissue. Aromatase enzyme is a transcription product of the CYP19 gene on chromosome 15q21 [12]. Promoters are identified in exon 1.4 in both osteoblastic and osteoclastic cells. The non-active mutation of aromatase gene results is associated with increased bone turnover and decreased BMD in both women and men [26].

In men, the androgen steroid hormone is dominant with possible implications in the maintenance of bone structure because hypogonadism determines the decrease in BMD and increased bone turnover. Studies also show that estrogen contributes to the regulation of bone growth and mineralization through aromatase activity in androgens that convert it in estrogen in peripheral tissues. Increased bone turnover and severe osteoporosis are found in men with homozygous CYP19 mutations [27].

\section{J. Osteocalcin}

Osteocalcin is one of the markers of specific bone metabolism activity produced by osteoblasts present in the organic bone matrix and used as a marker of bone formation activity. Osteocalcin is a specific protein that can be detected through ELISA examination [28]. In postmenopausal women, there will be an increase in the number of osteoclasts equal to the increase in the number of osteoblasts that play a role in the bone formation process along with the resorption process resulting in decreased bone mineral density. Osteocalcin is a specific protein matrix in bone that is primarily produced by bone osteoblasts that begin in the latent phase of the bone formation process [23,14]. Osteocalcin is a marker of the bone formation process. Most of the osteocalcin is attached to the part of the bone that will undergo the process of bone formation and a small part is secreted into the blood circulation so that it can be detected in the blood serum through immunoassay examination [20]. Osteocalcin is a marker of the bone formation process. Most of the osteocalcin is attached to the part of the bone that will undergo the process of bone formation and a small part is secreted into the blood circulation so that it can be detected in blood serum through immunoassay examination [28].

\section{K. Leptin}

Leptin is a hormone formed by fat cells that are released in the blood, the amount of leptin released in the blood depends on the amount of body fat present. Leptin is then taken to the brain and binds to the neurons in the hypothalamus [29]. One of the effects of leptin is a lack of appetite and increases the usefulness of body energy. Obesity is sometimes caused by resistance to the appetite-lowering effects of leptin.
People who are overweight tend to not experience osteoporosis for a long time and are not known why. Lately, there has been an association between leptin and decreased bone mass [30].

\section{Interferon beta (inf $\beta$ )}

Tadatsugu \& Taniguchi present evidence of interferon beta involvement in osteoclast differentiation [23]. They present evidence that osteoclasts can have an effect on their own differentiation and function on negative feedback mechanisms. The transcription of calcium phosphatase factor activated by RANKL has long been known. Taniguchi believes that calcium phosphatase can directly activate the expression of the gene [22]. Interferon beta can cause decreased levels of calcium phosphatase, thus pressing the osteoclast function [31].

\section{Thyroid hormone}

The thyroid gland, which looks like a butterfly, produces the hormone thyroxine that serves to maintain optimal metabolic rate of tissue for the normal functioning of the cell and the body as a whole. Thyroxine containing iodine (I) stimulates the consumption of $\mathrm{O} 2$ in the body cells, and regulates the metabolism of fats and carbohydrates necessary for growth and normal development of the body [22]. The function of the thyroid gland is controlled by the trophic hormone TSH (thyroid stimulating hormone or thyrotropin) secreted by the anterior pituitary gland. Conversely this tropical hormone secretion is also controlled in part by TRH (Thyrotropin releasing hormone) secreted by the hypothalamus. Some are also controlled by negative feedback mechanisms by the circulating free thyroxin hormone in the blood that inhibits the work of the anterior pituitary and hypothalamus $[9,13]$. Through this path, changes in the inner and external environment of the body will cause an adjustment to the secretion of thyroxine hormones in the form of T4 (tetraiodotironin) and T3 (triiodotironin). Except for producing thyroxin, the thyroid gland also produces the hormone calcitonin, a hormone whose effect lowers blood calcium levels [31].

\section{N. Growth factor}

The growth factor is a protein involved in cell replication, differentiation and function. Many of them have an important role in bone. Among them are Insulin -Like Growth Factor-I (IGF-I) and II (IGF-II) - both involved in bone formation and Transforming Growth Beta Factor (TGF-B) -insociated in bone formation and resorption [20,32].

\section{DISCUSSION}

Molecular evidence of immunologic mediation factors supports an explanation of the association of osteoporosis and inflammation [1]. This association is demonstrated by high-sensitivity (hs) CRP (Creative Protein) levels and bone mineral density in some inflammatory diseases. Albright about 65 years ago 
states that there is a relationship of physiological estrogen degradation in premenopausal, postmenopausal and postmenopausal age, leading to decreased osteoblast activity and increased osteoclast activity [19]. As a result the bone is resorbed by osteoclasts without being completely re-formed by the osteoblasts so primary osteoporosis occurs at postmenopausal age [13].

The occurrence of osteoporosis is highly dependent on the peak of bone mass that is strongly influenced by genetic factors. It also depends on calcium intake, physical activity, sun exposure, lifestyle (alcohol, cigarettes) and medications (corticosteroids, hormonal contraceptives) and estrogen hormone status during reproductive periods (menstrual regularity). Both women and men entering the second decade of life, there will be a loss of bone mass of $0.3-0.5 \%$ per year, especially in women of premenopausal age in whom estrogen levels decreased so that the loss of bone mass can reach up to 10-fold [19].

As the conclusion, the occurrence of osteoporosis is highly dependent on the peak of bone mass that is strongly influenced by genetic factors. Strong evidence suggests that decreased endocrine function, particularly estrogen secretion in menopausal women, results in increased bone resorption rate. The role of genes and hormones in the process of bone formation and resorption metabolism affect the occurrence of fractures in patients with osteoporosis

\section{REFERENCES}

[1] G. Jonasson, M. Rythen, "Alveolar bone loss in osteoporosis: a loaded and celluler affair?" Clinical, Cosmetic and Investigational Dentistry, vol. 8, pp. 95-103, 2016.

[2] G. Zhai, T. Andrew, et al., "Genetic and environmental determinants on bone loss in postmenopausal Caucasian women: a 14-year longitudinal twin study," Osteoporos Int., vol. 20, pp. $949-953,2009$.

[3] L.Z.G. Touyz, "Osteoporosis and oral implications," J. Osteopor Phys. Act, vol. 2(2), pp. 119, 2014.

[4] D.A. Atwood, "Some clinical factors related to rate of resorption of residual ridges," J. Prosthet. Dent., vol. 86, pp. 119-125, 2001.

[5] F.E. Ardakani, S.J. Mirmohamadi, "Osteoporosis and oral bone resorption: A review," J. Maxillofac. Oral Surg., vol. 8(2), pp. 121-126, 2009.

[6] S.C. Manolagas, "Birth and death of bone cells: Basic regulatory mechanism and implications for the pathogenesis and treatment of osteoporosis," Endocrine Reviews, vol. 21, pp. 115-137, 2000.

[7] S.L. Teitelbaum, F.P. Ross, L.S. Teitelbaum S, P.F. Ross, "Genetic regulation of osteoclast development and function," Nat. Rev. Gen., August 2003.

[8] S. Noordin, J. Glowacki, "Parathyroid hormone and its receptor gene polymorphisms:implications in osteoporosis and in fracture healing," Rhematol. Int., vol. 36, pp. 1-6, 2016.

[9] M. Bellana, M. Pirisi, P.P. Sainaghia, "Osteoporosis in rheumatoid arthritis: role of the vitamin D/parathyroid hormone system," Rev. Bras. Reumatol., vol. 55(3), pp. 256-263, 2015.

[10] L. Osagie-Clouard, A. Sanghani, M. Coathup, et al., "Parathyroid hormone 1-34 and skeletal anabolic action the use of parathyroid hormone in bone formation, Bone Joint," Res., vol. 6, pp. 14-21, 2017.

[11] C.J. Gruber, W. Tschugguei, C. Schneebeger, J.C. Huber, "Production and action of estrogens," N. Eng., J. Med, vol. 346, pp. 340-50, Jan 2002.
[12] J.J. Wysolmerski, "Parathyroid hormone-related protein: An update,” J. Clin. Endocrinol. Metab., vol. 97, pp. 2947-2956, 2012.

[13] M.J. Seibel, "Biochemical markers of bone turnover part I: biochemistry and variability," Clin. Biochem. Rev., vol. 26, pp. 97-122, 2005.

[14] A. Maedaa, M. Okazakia, et al., "Critical role of parathyroid hormone $(\mathrm{PTH})$ receptor-1 phosphorylation in regulating acute responses to PTH,” PNAS., vol. 110(15), pp. 5864-5869, April 2013.

[15] N.M. Al-Daghri, O.S. Al-Attas, et al. "Association between promoter region genetic variants of PTH SNPs and serum 25(OH)-vitamin D level,” Int. J. Clin. Exp. Pathol., vol. 8(7), pp. 8463-8471, 2015.

[16] S.C. Manolagas, S. Kousteni, R.L Jilka, "Sex steroids and bone," The Endocrine Society, 2002.

[17] O. Bell, J. Silver, T. Naveh-Many, Parathyroid hormone, from gene to protein. Molecular biology of the parathyroid, Eurekah Comand Kluwer Academic/Plenum Publishers, 2005, 8-30.

[18] D.G. Monroe, F.J. Secreto, T.C Spelsberg, "Overview of estrogen action in osteoblasts: Role of the ligand the receptor and the co-regulators," J. Musculoskel. Neuron. Interact., vol. 3(4), pp. 357-62, 2003.

[19] O.I Kurt, H. Yilmaz-Aydogan, et al., "Evaluation of ER $\alpha$ and VDR gene polymorphisms in relation to bone mineral density in Turkish postmenopausal women,” Mol. Biol. Rep., vol. 39(6), pp. 6723-6730, June 2012.

[20] S. Lindawati, I. Kusdhany, E. Auerkari, A. Dwi, W. Suryandari, Tribudi, I. Rahardjo, E. Hogervoorst, C. Talbot, "Estrogen receptor $\beta$ gene polymorphisms and osteoporosis risk in postmenopausal women in need of dentures," International Journal of Clinical Preventive Dentistry, vol. 7(1), March 2011.

[21] Bell, H. Norman, "RANK ligand and the regulation of skletal remodeling”, J. Clin. Invest., vol. 111, pp. 1120-22, 2003.

[22] X.J. Xu, L. Shen, et al., "Serum $\beta$-Catenin levels associated with the ratio of RANKL/OPG in patients with postmenopausal osteoporosis," International Journal of Endocrinology, vol. 7, 2013

[23] D. Savitsky, T. Tamura, H. Yanai, T. Taniguchi, "Regulation of immunity and oncogenesis by the IRF transcription factor family," Cancer Immunology, Immunotherapy, vol. 59(4), pp. 489-510, April 2010

[24] A.E. Kearns, S. Khosla, P.J. Kostenuik PJ, "Receptor activator of nuclear factor $\mathrm{kB}$ ligand and osteoprotegerin regulation of bone remodeling in health and disease," Endocrine Reviews, vol. 29(2), pp. 155-92, 2008.

[25] M. Augustine, M.J. Horwitz, "Parathyroid hormone and parathyroid hormone-related protein analogs as therapies for osteoporosis," Curr. Osteoporos. Rep., vol. 11, pp. 400-406, 2013.

[26] A.M. Elmesiry, "Osteoporosis: Mineral metabolism and arthritis,” J. Osteopor. Phys. Act., vol. 4(1), pp. 1, 2015.

[27] X.Y. Zhang, J.W. He, et al., "Associations of serum osteocalcin and polymorphisms of the osteocalcin gene with bone mineral density in postmenopausal and elderly chinese women," J. Nutrigenet. Nutrigenomics, vol. 9, pp. 231-242, 2016.

[28] P Aggarwal, A. Zavras, "Parathyroid hormone and its effects on dental tissues," Oral Diseases, vol. 18, pp. 48-54, 2012

[29] I. Zofkova, P. Nemcikova, M. Kuklik, "Polymorphisms associated with low bone mass and high risk of atraumatic fracture," Physiol. Res., vol. 64, pp. 621-631, 2015.

[30] F. Rivadeneira, O. Mäkitie, "Osteoporosis and bone mass disorders: From gene pathways to treatments," Trends in Endocrinology \& Metabolism.

[31] J. Phetfong, T. Sanvoranart, "Osteoporosis: the current status of mesenchymal stem cell-based therapy," Cellular \& Molecular Biology Letters, vol. 21, pp. 12, 2016.

[32] G.Y. Rochefort, "The osteocyte as a therapeutic target in the treatment of osteoporosis", Ther. Adv. Musculoskel. Dis., vol. 6(3), pp. 79-91, 2014.

[33] M.J. Oursler, "Direct and indirect effects of estrogen on osteoclast,” J. Musculoskel. Neuron Interact., vol. 3(4), pp.363366, 2003. 